\title{
Unpacking vulnerability: towards language that advances understanding and resolution of social inequities in public health
}

\section{Décomposer la vulnérabilité : vers un langage qui favorise la compréhension et la résolution des iniquités sociales en santé publique}

\author{
Lindsay McLaren ${ }^{1}$ - Jeff Masuda ${ }^{2}$ - Janet Smylie ${ }^{3} \cdot$ Christina Zarowsky $^{4,5}$ \\ Published online: 28 January 2020 \\ (C) The Canadian Public Health Association 2020
}

Our attention was drawn to an important recent paper published in the journal Critical Public Health, which discusses the use (and misuse) of the word "vulnerable" in public health research and practice (Katz, Hardy, Firestone, Lofters, \& Morton-Ninomiya, 2019). We commend these authors for contributing a timely paper that calls attention to the role of language in what we regard as the longstanding challenge of downstream drift in public health research (e.g., Baum \& Fisher, 2014; Baum \& Sanders, 2011; Carey, Malbon, Crammond, Pescud, \& Baker, 2016).

The paper caught our eye, in part, because the authors went about their task by identifying and analyzing recent articles published in the Canadian Journal of Public Health (CJPH), as well as the American Journal of Public Health, that had used the word "vulnerable" in a way that was "vaguely, inconsistently or undefined at least once." These two journals, which are aligned with the national public health associations in Canada and the United States, respectively, were viewed as illustrative of the ways in which phrases such as "vulnerable population" are used in mainstream public health discourse in the two countries.

Lindsay McLaren

lmclaren@ucalgary.ca

University of Calgary, Calgary, AB, Canada

2 Queen's University, Kingston, ON, Canada

3 University of Toronto and St. Michael's Hospital, Toronto, ON, Canada

4 Université de Montréal, Montréal, QC, Canada

5 University of the Western Cape, Cape Town, South Africa
Using critical discourse analysis, Katz et al. (2019) explored articles that used the term "vulnerable" and highlighted examples - within both journals — of papers where phrases such as "vulnerable groups" or "vulnerable populations" were defined vaguely, or not at all. In such circumstances, the authors argue, the reader is left to "'fill in the blanks' as to who is vulnerable, why they are vulnerable, and what they are vulnerable to." This in turn can have the effect of obscuring systemic and structural causes of inequity, including the role of power, and can perpetuate a focus on mechanisms and interventions that are downstream in orientation (Katz et al., 2019).

For many readers of the CJPH, these insights are not new. From a global public health perspective, for example, there is a rich legacy of work that aims for a more thoughtful and sophisticated understanding of vulnerability as both a condition and a set of processes (Zarowsky, Haddad, \& Nguyen, 2013). Important work in the area of Indigenous health and well-being has identified that an uncritical lens on vulnerability can lead to a deficit discourse, or language that emphasizes deficiencies, rather than strengths, of communities (Smylie, Lofters, Firestone, \& O'Campo, 2012). Recent work in this journal, in a special section on the topic of Why public health matters today, considers how a population-level approach to prevention - arguably a foundation of public health - can be one way of maintaining a focus on the structures and processes that create and perpetuate vulnerability in the first place (McLaren, 2019).

Yet, as Katz et al. (2019) demonstrate, simplistic and problematic use of the concept of vulnerability persists. Why? As these authors note, researchers sometimes use the word "vulnerable" strategically, to attract resources, policy interest, and public concern (commendably, the authors include themselves within that statement). As editorial board members of an academic 
journal, it would be remiss of us not to acknowledge that journal word limits can place constraints around the extent to which authors can thoughtfully articulate complex concepts like vulnerability. Within such circumstances, the practice of taking linguistic shortcuts - such as using single adjectives to describe a complex set of processes and circumstances - is something that many of us do.

One of Katz et al.'s (2019) important findings was that, among the articles they explored, "few papers examined the flip side of 'vulnerable populations' - the individuals and groups that are not considered to be vulnerable"; moreover, they identified that "While researchers often describe the policy landscapes, legislation and processes that lead to poor health outcomes, they do not generally examine the individuals and groups enacting policy decisions, or the factors that incentivize, enable, connect and facilitate the individuals and groups producing vulnerability" (italics added). Indeed, there are many examples of research funding competitions focused on "vulnerable" populations (e.g., NIH , 2019; University of Calgary, 2019); why aren't funding calls in public health instead organized around political structures, and those who create and perpetuate inequity?

Policing the use of language is outside of the remit of our role as journal editors. However, it behooves us at the Canadian Journal of Public Health to try to be part of the solution, rather than part of the problem. Our explicit acknowledgement of these issues, in this editorial, is one attempt to do so. More broadly, we pose the question, and challenge, to the CJPH community, including research funders: How do we effectively situate our work within, or against, existing power structures and relations between those who are oppressed and the ostensible oppressors? How do we avoid perpetuating the status quo of a deficit-based mentality in public health?

Lindsay McLaren, Jeff Masuda, Janet Smylie, Christina Zarowsky

Senior Editors, CJPH

\section{Éditorial}

Notre attention a été attirée vers un important article, publié récemment dans la revue Critical Public Health, qui traite de l'usage (et du mésusage) du mot «vulnérable » dans la recherche et la pratique en santé publique (Katz et al., 2019). Nous félicitons les auteurs d'avoir contribué un article d'actualité sur le rôle du langage dans ce que nous jugeons être un problème de longue date : la « dérive vers l'aval» de la recherche en santé publique (voir p. ex. Baum \& Fisher, 2014; Baum \& Sanders, 2011; Carey et al., 2016).

L'article a retenu notre attention, en partie, car les auteurs s'y sont pris en répertoriant et en analysant des articles parus récemment dans la Revue canadienne de santé publique
(RCSP) et dans l'American Journal of Public Health où le mot « vulnérable » était employé « au moins une fois de manière vague, contradictoire ou non définie » [traduction libre]. Les deux revues, qui relèvent des associations nationales pour la santé publique du Canada et des États-Unis, respectivement, étaient jugées illustrer les façons dont des expressions comme « populations vulnérables » sont employées dans le discours dominant sur la santé publique dans les deux pays.

$\mathrm{Au}$ moyen d'une analyse critique du discours, Katz et collègues ont exploré les articles où le mot « vulnérable » était employé et relevé des exemples - dans les deux revues d'articles où des expressions comme " groupes vulnérables » ou « populations vulnérables » n'étaient pas définies, ou trop vaguement. Dans ces situations, les auteurs font valoir qu'on laisse le soin au lecteur de « combler les trous » en déterminant qui est vulnérable, pourquoi ces gens sont vulnérables et à quoi ils sont vulnérables. En retour, cela peut avoir pour effet d'obscurcir les causes systémiques et structurelles des iniquités, dont le rôle du pouvoir, et de perpétuer l'attention accordée aux mécanismes et aux interventions en aval (Katz et al., 2019).

Pour bien des lecteurs de la RCSP, ces idées ne sont pas nouvelles. Du point de vue de la santé publique mondiale, par exemple, il existe un riche héritage d'études qui visent à une compréhension plus réfléchie et plus complexe de la vulnérabilité en tant que condition et en tant qu'ensemble de processus (Zarowsky et al., 2013). Selon un article important sur la santé et le bien-être des peuples autochtones, une optique non critique de la vulnérabilité peut mener à un discours de déficit ou à un langage centré sur les carences des communautés plutôt que sur leurs forces (Smylie et al., 2012). Un article publié récemment dans une rubrique spéciale de la présente revue sur le thème de Pourquoi la santé publique aujourd'hui? cherche à savoir comment une approche de prévention populationnelle - qui est probablement l'une des bases de la santé publique - peut être un moyen de maintenir l'attention sur les structures et les processus qui créent et qui perpétuent la vulnérabilité pour commencer (McLaren, 2019).

Pourtant, comme le démontrent Katz et collègues (Katz et al., 2019), l'usage simpliste et problématique de la notion de vulnérabilité persiste. Pourquoi? Comme l'indiquent les auteurs, les chercheurs emploient parfois le mot « vulnérable » de façon stratégique, pour attirer des ressources et l'intérêt des décideurs et du public (les auteurs s'incluent à raison dans cet énoncé). Il serait négligent de notre part, nous les membres du comité éditorial d'une revue savante, de ne pas reconnaître que les limites de mots imposées par ces revues peuvent empêcher les auteurs d'expliquer à fond des notions aussi complexes que la vulnérabilité. Dans ces circonstances, la pratique qui consiste à emprunter des raccourcis linguistiques - comme l'emploi d'adjectifs uniques pour décrire un jeu complexe de processus et de situations - est une chose que nous sommes nombreux à faire. 
L'une des grandes constatations de Katz et collègues est que de tous les articles qu'ils ont explorés, rares sont ceux qui portent sur l'opposé des «populations vulnérables »- les personnes et les groupes qui ne sont pas jugés vulnérables; de plus, bien que les chercheurs décrivent souvent les politiques globales, les lois et les processus qui mènent à la détérioration de la santé, ils n'examinent pas en général les personnes et les groupes qui prennent les décisions stratégiques, ni les facteurs qui encouragent, habilitent, mettent en rapport et favorisent les personnes et les groupes qui produisent la vulnérabilité (les italiques sont de nous). Il y a à vrai dire de nombreux exemples de concours de financement d'études sur les populations « vulnérables » (p. ex. National Institutes of Health, 2019; Université de Calgary, 2019); pourquoi les appels de financement en santé publique ne s'articulent-ils pas plutôt autour des structures politiques, et de ceux qui créent et qui perpétuent les iniquités?

Le contrôle de l'usage du langage ne fait pas partie de notre rôle de rédacteurs de revues. Il nous incombe cependant, à la Revue canadienne de santé publique, d'essayer de faire partie de la solution plutôt que du problème. Notre reconnaissance explicite de ces problèmes, dans le présent éditorial, est une tentative en ce sens. Plus généralement, nous avons une question à poser (et un défi à lancer) à la communauté de la RCSP, y compris aux bailleurs de fonds de recherche : Comment situons-nous dans les faits notre travail, en phase ou en opposition avec les structures et les relations de pouvoir existantes entre les opprimés et les oppresseurs apparents? Comment évitons-nous de perpétuer le statu quo d'une mentalité déficitaire en santé publique?

Lindsay McLaren, Jeff Masuda, Janet Smylie, Christina Zarowsky

Rédacteurs scientifiques adjoints, RCSP

\section{References/Références bibliographiques}

Baum, F., \& Fisher, M. (2014). Why behavioural health promotion endures despite its failure to reduce health inequities. Sociology of Health \& Illness, 36(2), 213-225.

Baum, F., \& Sanders, D. M. (2011). Ottawa 25 years on: a more radical agenda for health equity is still required. Health Promotion International, 26(suppl 2), ii253-ii257.

Carey, G., Malbon, E., Crammond, B., Pescud, M., \& Baker, P. (2016). Can the sociology of social problems help us to understand and manage 'lifestyle drift'? Health Promotion International, 32(4), 755-761.

Katz, A. S., Hardy, B.-J., Firestone, M., Lofters, A., \& Morton-Ninomiya, M. E. (2019). Vagueness, power and public health: use of 'vulnerable' in public health literature. Critical Public Health. https://doi. org/10.1080/09581596.2019.1656800. (online first)

McLaren, L. (2019). In defense of a population-level approach to prevention: why public health matters today. Canadian Journal of Public Health, 110(3), 279-284.

National Institutes of Health (NIH). (2019). Call for submissions on "Healthy Habits: Timing for Developing Sustainable Healthy Behaviors in Children and Adolescents" which includes "identifying factors that support healthy behavior development in vulnerable populations" (https://grants.nih.gov/grants/guide/pa-files/PA-18355.html).

Smylie, J., Lofters, A., Firestone, M., \& O'Campo, P. (2012). Populationbased data and community empowerment. In P. O'Campo \& J. R. Dunn (Eds.), Rethinking Social Epidemiology: Towards a Science of Change (pp. 67-92). London: Springer.

University of Calgary, O’Brien Institute for Public Health. (2019). Special catalyst competition: socially vulnerable populations (https:// obrieniph.ucalgary.ca/CatalystFundingandEventSponsorship)

Zarowsky, C., Haddad, S., \& Nguyen, V.-K. (2013). Beyond 'vulnerable groups': contexts and dynamics of vulnerability. Global Health Promotion, 20(Supp. 1), 3-9.

Publisher's note Springer Nature remains neutral with regard to jurisdictional claims in published maps and institutional affiliations. 